\title{
Transparencia, razón pública y rendición de cuentas en las empresas
}

\author{
WILSON HERRERA* \\ Universidad del Rosario (Colombia) \\ wilson.herrera@urosario.edu.co \\ IVAN MAHECHA** \\ Universidad del Rosario (Colombia) \\ imahecha@gmail.com
}

\begin{abstract}
Resumen
Este artículo versa sobre la relación entre rendición de cuentas ética y transparencia en el marco de la ética empresarial. Se argumenta que a la rendición de cuentas le debe ser inherente la transparencia con el fin de que una auditoría sea verdaderamente ética y no un simple medio de incrementar la reputación ética empresarial. Para ello, se analiza el concepto de transparencia, visto desde la ética cívica, y cómo este se implica en un enfoque normativo de la teoría de los stakeholders. Se finaliza abordando algunos parámetros mínimos de transparencia que la rendición de cuentas debe tener para que las empresas que los apliquen puedan ser efectivamente consideradas como empresas justas.
\end{abstract}

Palabras clave: Transparencia, rendición de cuentas, ética cívica, ética empresarial, stakeholders.

\section{Transparency, public reason and accountability in companies}

\begin{abstract}
This article revolves around the relationship between ethical accountability and transparency within the framework of business ethics. It is argued that, for the purpose of an audit to be really ethical rather than merely a tool to increase the levels of the reputation of the company, transparency must be inherent to accountability. To do this, it is claimed that the concept of transparency is analyzed should be seen from the point of view of civic ethics, in order to establish how it is implied in a normative approach to stakeholders theory. The article ends arguing that any process of accountability must include some minimal normative components of transparency for it to be applied in companies wanting to be considered as fair companies.
\end{abstract}

Key words: Transparency, accountability, civic ethics, Business ethics, stakeholders.

* Doctor en Filosofía (Binghamton University). Profesor Titular de la Escuela de Ciencias Humanas de la Universidad del Rosario. Miembro del Grupo de Ética Aplicada, trabajo y cambio social. Director del Centro de Formación en Ética y Ciudadanía, Phronimos.

** Doctorando de Derecho de la Universidad del Rosario en Colombia y de la Universidad de Tilburg en los Países Bajos. Joven Investigador del Centro de Formación en Ética y Ciudadanía, Phronimos, y miembro del Grupo de Ética Aplicada, trabajo y cambio social. 


\section{INTRODUCCIÓN}

La transparencia, y las políticas anticorrupción que se le asocian, tiene, en principio, un doble propósito en un estado social de derecho: garantizar que los recursos públicos se usen de manera eficiente en la satisfacción de las necesidades de los ciudadanos y contribuir a que la deliberación pública no se distorsione por las presiones de intereses particulares. En lo que concierne a las empresas productivas, la transparencia y la rendición de cuentas están ligadas a dos propósitos: proveer los bienes y servicios requeridos por los ciudadanos y generar beneficios económicos a los propietarios de las empresas. En relación con lo primero, la transparencia es un valor asociado a la eficiencia de las empresas y apunta al buen funcionamiento de los mercados; suponiendo, claro está, que los mercados son competitivos e incentivan la eficiencia. En cuanto al segundo, la transparencia busca garantizar que los propietarios reciban las utilidades a que tienen derecho.

Más allá de estas dos justificaciones, la transparencia en las empresas tiene un tercer propósito, más fundamental: tal como acontece con el Estado, las empresas productivas, especialmente las más grandes, tienen un inmenso poder sobre los distintos actores sociales ${ }^{1}$. Asociada con este poder, la transparencia tiene que ver con el control que se ejerce sobre estas organizaciones. En otras palabras, la transparencia tiene el papel de defender al sistema político democrático y al sistema económico de los abusos de poder que pueden ejercer las empresas. Prueba de esta interferencia son los casos de corrupción más sonados en Colombia, que han distorsionado los procesos democráticos y desviado recursos valiosos para satisfacer los intereses de ciertos grupos empresariales. En este sentido, transparencia y rendición de cuentas están íntimamente conectadas².

Desde esta perspectiva, no sobra recordar que las empresas son organizaciones complejas en las que hay conflictos entre los distintos grupos

1 De acuerdo con Joseph Lozano, las empresas no solo tienen poder económico, también tienen la capacidad de afectar significativamente las identidades culturales, las relaciones sociales entre grupos e individuos, el régimen político, el desarrollo de la ciencia y la tecnología y el medio ambiente (Lozano, 1999: 120-121).

2 En las discusiones contemporáneas sobre ética empresarial es común que la auditoria ética y la rendición de cuentas se usen como si fueran una y la misma cuestión. Sin embargo, también es común que se indique que la rendición de cuentas es solo una etapa de los procesos de auditoria ética: la de comunicación y retroalimentación (ver supra 2.4). En este artículo, rendición de cuentas y auditoria ética son conceptos intercambiables, con la salvedad de que, para ser considerada sinónimo de auditoria ética, la rendición de cuentas debe cumplir con los requisitos que se indicarán en la segunda parte del artículo. Así, no toda rendición de cuentas puede considerarse auditoria ética, sino solamente aquella que cumpla los requisitos normativos necesarios para ello. 
involucrados en su funcionamiento. En un enfoque normativo de la teoría de los stakeholders que, además, atiende a principios democráticos, en el proceso de auditoria ética deben participar todos los stakeholders afectados y diseñarse para que las presiones indebidas por parte de los grupos más influyentes no tengan cabida. Desde nuestro punto de vista, lo que emerge de lo anterior es que un proceso de rendición de cuentas ética no puede estar bajo el control exclusivo del grupo de interés más poderoso; de otro modo, no puede decirse que dicho proceso es transparente.

Ahora, si a la transparencia le es inherente la rendición de cuentas, surge la cuestión de cómo las organizaciones deben llevar a cabo este proceso de forma transparente en el marco de un estado social de derecho. Para responder esta interrogante, a lo largo del trabajo se hará un análisis conceptual de lo que significa la transparencia en el marco de una ética cívica y, a partir de allí, se discutirá cómo esta puede permear a una empresa que tenga la pretensión de responder a los valores que configuran los valores de un régimen democrático y que tiene como fin garantizar los derechos y libertades de los ciudadanos.

Las reflexiones que se presentan a continuación no se hacen aisladas de la realidad empresarial. Por tal motivo, en el trabajo se tienen como referente dos iniciativas de estándares sociales y éticos, el GRI y el ETHOS, que han tenido cierta influencia en número importante de empresas latinoamericanas, incluidas varias colombianas. Para dar cuenta de la pregunta planteada, este artículo tiene la siguiente estructura: en primer lugar, nos detendremos en el concepto de transparencia (1) y sus relaciones con el concepto de razón pública (1.1) y con otros valores (1.2). Posteriormente, abordaremos el tema de los stakebolders y la rendición de cuentas (2). Comenzamos con una breve presentación de la teoría de los stakeholders (2.1), y discutimos cuestiones centrales que, según este enfoque, deben tenerse en cuenta en la manera cómo las empresas deben rendir cuentas a los grupos afectados por sus actividades: ¿cuáles son los grupos de interés que deben considerarse? (2.2); ¿cuáles intereses legítimos deben tenerse en cuenta? (2.3); y ¿cuál es el papel de los stakeholders en la rendición de cuentas? (2.4). Finalmente, el artículo buscará establecer unos mínimos de transparencia que deben ser considerados en el proceso de rendición de cuentas por parte de las empresas que tengan la pretensión de ser organizaciones justas (3).

\section{TRANSPARENCIA}

De acuerdo con Transparencia Internacional (2018), la transparencia es el mecanismo más seguro para combatir la corrupción y ayudar a incre- 
mentar la confianza en los funcionarios y las instituciones públicas; la corrupción, por su parte, es el abuso del poder que se le ha encomendado a alguien con el fin de obtener provecho propio. Transparencia es iluminar las reglas, planes, procesos y acciones, así como saber el porqué, cómo, qué y cuánto de las decisiones, esto con el fin de asegurar que los funcionarios públicos, directivos, y empresarios actúen de forma razonable y visible en el reporte de sus actividades. Así, la transparencia se erige como el mejor instrumento para que el público en general pueda seguir las acciones de aquellos que tienen en sus manos la toma de decisiones; para poder hacerlos responsables de sus acciones; y finalmente, para que no abusen de su poder a fin de obtener provecho propio. A la transparencia, entendida de esta manera, le es inherente el acto de rendir cuentas.

Cobra sentido, entonces, que a la pregunta sobre por qué las empresas deberían ser transparentes se responda que ser transparentes (rendir cuentas) genera mayor confianza ${ }^{3}$. Si se pregunta nuevamente acerca del por qué la empresa debería generar mayor confianza, invariablemente la respuesta se relaciona, de un modo u otro, con la eficiencia y la productividad (como valores últimos que no requieren ser fundamentados). Esta conclusión se apalanca en los argumentos tradicionales que se tienen en cuenta respecto del por qué las empresas deberían actuar éticamente (Entrepreneur, 2017; Mundo Ejecutivo, 2017; Forbes, 2018).

De este modo, el requerimiento de las empresas por ser éticas, es decir transparentes, y por ende la exigencia de rendir cuentas, tiene un carácter instrumental y necesariamente ligado a la eficiencia empresarial. Si las empresas no son transparentes, incurren en costos altos debido a la falta de confianza que los trabajadores, los consumidores y la sociedad en general tiene respecto de ella. Estos costos agregados, que son parte de lo que Ronald Coase $(1937 ; 1960)$ denominó costos de transacción, impactan fuertemente la inversión que debe hacerse en los rubros de negociación y decisión, y de vigilancia y ejecución (Dahlman, 1979), algo que incide negativamente en la eficiencia de la empresa. En cambio, una empresa transparente es una organización más confiable, lo que es una alternativa menos costosa frente, por ejemplo, al uso de instrumentos de vigilancia coercitivos e invasivos.

\footnotetext{
3 Esta idea se deriva del siguiente argumento: si la corrupción se relaciona con obtener provecho propio, que es precisamente el fin esencial de las empresas, y la transparencia lucha contra las actividades que en el sector público buscan la obtención de provecho propio, ¿por qué las empresas deberían hacer lo contrario a aquello que les genera provecho propio?
} 
Así, la transparencia y la confianza entran en una relación instrumental que sólo tiene sentido si se tiene a la eficiencia como horizonte: si el objetivo de la empresa es ser eficiente en la generación de ganancia, una de las acciones que debe emprender es la reducción de los costos de transacción; si dicha reducción puede hacerse al generar más confianza en la sociedad y si dicha confianza puede generarse a través del ejercicio de la transparencia, entonces, es lógico que la empresa deba ser, asimismo, transparente.

La desventaja obvia de este argumento es que está supeditado al cálculo del beneficio económico del ejercicio empresarial. Esto es, si al hacer el cálculo se encuentra que se es más eficiente en la generación de ganancias incluyendo los costos de transacción relacionados con la corrupción, la necesidad de ser transparentes desaparece. Por supuesto, en este cálculo debe incluirse la posibilidad de la pérdida de confianza respecto de la empresa: bien puede ser que dicha pérdida ya esté contemplada en los necesarios costos de transacción y que, de todas maneras, la generación de ganancias siga siendo positiva, o que se diseñen medios para evitar la pérdida de confianza sin que necesariamente se eviten las prácticas corruptas que generan ganancias.

Existe otra forma de entender la transparencia en el contexto empresarial, la cual se centra, por un lado, en abogar por una definición que integre la ética de lo público (o ética cívica) y, por otro, en relativizar la idea de que la transparencia es el único instrumento en la lucha contra la corrupción. Para ello, partimos de una concepción normativa de la transparencia, que la asume necesariamente conectada con la democracia; esta última, siguiendo la propuesta de John Dewey (1951: 394), debe ser entendida no como un mero régimen político, sino como una forma de vivir juntos, que es pluralista e incluyente, y en la que se prometen y promueven, entre otros valores, la autonomía, la equidad y la solidaridad de los ciudadanos.

En su relación con la democracia, la transparencia ha sido vinculada tradicionalmente con el ejercicio de las funciones públicas, hasta el punto en el que esta sólo tiene relevancia para el sector privado cuando se conecta con temas como la eficiencia y la productividad. Así las cosas, siguiendo la lectura de Transparencia Internacional (2018), la transparencia descansa en la prevención de los costos derivados de la corrupción, a saber: costos políticos, relacionados con la pérdida de legitimidad de la democracia y el estado de derecho de los sistemas políticos corruptos; costos económicos que impactan la riqueza del país cuando los servidores públicos utilizan los recursos para incrementar su riqueza personal; costos sociales, al disminuir la confianza de los ciudadanos en su sistema político, sus instituciones y líderes; y costos medioambientales, cuando las instituciones públicas no regulan la explotación de las riquezas nacionales o, más usualmente, los 
funcionarios evitan aplicar la legislación debido a la recepción de sobornos.

Así entendida, la corrupción tendría consecuencias dañinas para la democracia, pero pareciera que sólo en tanto dichas consecuencias afecten el ejercicio empresarial, las empresas deberían propender a combatirla. Un análisis detallado daría a entender que la transparencia es algo eminentemente público; es decir, algo que debe llevarse a cabo por parte de los funcionarios y entidades públicas y que sólo afecta a los privados en cuanto hay algunas consecuencias en lo público. En otras palabras, la transparencia en una democracia solamente tendría sentido para las organizaciones privadas en cuanto medio para la obtención de otros fines que son vistos como superiores (eficiencia y productividad).

Pero la transparencia no sólo es un medio para que la empresa gane en eficiencia y productividad; ella puede ser una expresión de que la empresa se toma en serio los intereses de los grupos afectados. Precisamente, una de las facetas de la confianza, o mejor, de traicionar la confianza, consiste en prometer que se van a considerar los intereses de una persona para luego actuar sin tenerlos en cuenta. Por el contrario, para lograr confianza, primero deben conocerse los intereses de la persona en cuestión y, luego, dar a conocer a dicha persona que sus intereses sí fueron tenidos en cuenta al tomar la decisión. Ello precisamente es transparencia: actuar razonable y visiblemente.

De este modo nos adentramos en una concepción de transparencia cuyo horizonte es la ética cívica, y en la cual la transparencia tiene que ver con el carácter público que debe tener un sistema ético dentro de una empresa, y no con la externalidad contingente propia de la productividad y la eficiencia. Es decir, una concepción cívica de la transparencia ya no la concibe como instrumental a la eficiencia y la productividad sino como un valor en sí mismo que debería ser promovido por las empresas sin necesidad de justificación externa. De este modo, la transparencia se justifica internamente; esto es, se relaciona necesariamente con dos aspectos sustantivos de un ethos democrático: por un lado, con la idea de razón pública (actuar razonable y visiblemente); y por otra, con otro tipo de valores que también hacen parte de una ética de lo público, tales como la autonomía, los derechos humanos, la equidad e, incluso, la eficiencia. Se trata, en otras palabras, de una concepción de la transparencia vinculada internamente con la democracia. 


\subsection{Respecto de la idea de razón pública}

Un uso instrumental de la transparencia es afín a una concepción utilitarista de la moral, predilecta en los contextos económicos y empresariales. En esta concepción, el criterio de decisión se deriva del principio utilitarista: una acción es justa si maximiza el bienestar del mayor número de personas; con lo cual, una acción es justa cuando se logra satisfacer más necesidades de los ciudadanos con el menor uso de recursos posibles. Así expresado, el principio utilitarista no es otro que el principio de la eficiencia.

No obstante, de acuerdo con John Rawls (1971), este principio tiene, desde un punto de vista moral, varios problemas, pero aquí solo destacaremos dos. En primer lugar, en el plano institucional es posible encontrar diferentes arreglos institucionales que sean igualmente eficientes; de acuerdo con esto, la eficiencia puede ser en ciertos contextos un criterio insuficiente de decisión y, por tanto, necesita ser complementado por otro. En segundo lugar, la aplicación del principio puede llevar a escoger alternativas que consideramos abiertamente injustas. Por ejemplo, según el criterio utilitarista, si un régimen dictatorial y opresivo genera un crecimiento mayor de la economía, deberíamos escoger dicho régimen frente a uno que garantice los derechos humanos, pero que no genera el mismo crecimiento económico.

Esta crítica muestra que el principio utilitarista no recoge ciertas intuiciones morales básicas que, al menos en sociedades que se dicen liberales y democráticas, son aceptadas por los ciudadanos, como, por ejemplo, la autonomía, la equidad o los derechos humanos; conceptos que en este tipo de escenarios tienen primacía sobre otro tipo de valores. En oposición a la concepción utilitarista, una concepción deontológica tiene como criterio fundamental la dignidad humana, principio según el cual, un curso de acción es justo si su máxima puede tener un carácter universal; es decir, si las razones para actuar pueden ser asumidas por cualquier agente moral.

Así, en reiteradas ocasiones Kant sostiene que, con respecto a las decisiones que afectan los derechos e intereses legítimos de los otros, una condición necesaria, aunque no suficiente, para que ellas sean justas es que al ser conocidas por todos no se destruya el propósito que pretenden alcanzar; dicho de otro modo, para Kant, una norma es justa si es capaz de pasar el escrutinio público ${ }^{4}$. El principio de la publicidad o transparencia exige, además, que las razones que motivan la decisión sean públicas. Siguiendo esta idea, John Rawls, en su análisis de la esfera pública en una

En el segundo apéndice de la Paz Perpetua, Kant afirma: “Todas las acciones que no sean compatibles con la publicidad son injustas” (2018: VIII/381). 
sociedad pluralista, apunta a que las razones que se esgrimen en el ámbito público deben ser basadas en creencias y métodos de indagación aceptados por todos, esto es, públicos (Rawls, 1999: 328).

Las variaciones más contemporáneas de las concepciones kantianas, apuntan al uso de la ética discursiva en el ámbito empresarial. El principio kantiano en clave discursiva lo ha formulado P. Ulrich (1987: 254) en los siguientes términos: "Una empresa podría considerarse ética cuando las decisiones, acciones y políticas que adopta, así como las consecuencias y efectos de las mismas respecto a los intereses en juego, pudieran ser aceptados por todos los implicados y/o afectados presentes y futuros en un diálogo abierto en condiciones simétricas de participación" (citado por García-Marzá, 2004: 158-159). En otras palabras: una empresa justa no se queda en la mera comunicación de sus decisiones; ya que abarca, además de la práctica de sustentar las decisiones y políticas en datos verificables y en principios morales compartidos, tener en cuenta los intereses de todos sus stakeholders de manera integral en su estructura y cultura organizativa. Estas acciones no son otra cosa que la aplicación del principio de transparencia en la práctica empresarial, lo cual pasa necesariamente por la debida construcción y justificación de indicadores de gestión ética y, en especial, de aplicación del valor de la transparencia en las empresas y su posterior seguimiento y evaluación. Precisamente, la segunda parte de este artículo se dedicará a las pautas para dicha construcción y justificación.

Tal vez una formulación más práctica para su aplicación al contexto empresarial sea la estructura del principio de justificación que realiza Rainer Forst (2011; 2014), el cual se deriva de la ética discursiva habermasiana, heredera de la tradición kantiana. Dicho principio reza que "cada norma que vaya a vincular a una pluralidad de personas (...) debe ser justificada con razones que sean recíprocamente aceptables por todos los afectados en tanto personas libres e iguales" (Forst, 2011: 20). Este principio discursivo implica un derecho moral básico a la justificación, a partir del cual todas las personas pueden rechazar razones de tipo particular usadas como justificación de las normas que se les aplican a todos y exigir que sean justificadas a partir de razones que se espera, razonablemente, que todos compartan o que, por lo menos, no sean razonablemente rechazadas.

Estas razones que se espera no sean rechazadas por los destinatarios de la norma se caracterizan por superar el denominado umbral de reciprocidad y generalidad. Este umbral establece el límite que debe cumplir la razón aportada para justificar una regla, estableciendo si la misma pertenece a un contexto de justificación en el cual se busca la satisfacción de intereses privados o a uno en el que los intereses de todos son tenidos en cuenta: si la razón aportada no supera el umbral, podemos determinar que 
la misma no es de carácter universal sino privado, por lo que no será adecuada para justificar una regla que vincule a una pluralidad de personas; sin embargo, dicha razón puede seguir siendo una razón adecuada para que la comunidad privada respectiva considere dicha norma como vinculante. Así, por ejemplo, una norma que regula la forma en que debe realizarse el pago a los empleados de una empresa, solo es justificable ante todos ellos si las razones dadas para dicha norma contemplan los intereses de todos sus empleados; pero si la razón solamente contempla los intereses de los directivos de la empresa, la norma no puede justificarse para todos los empleados sino solamente para los directivos.

Aunque ni Kant, Rawls, Habermas o Forst han considerado a las empresas en sus reflexiones, la exigencia de transparencia para adoptar una decisión puede serles aplicables en la medida en que la pretensión de ser justas requiere que los stakeholders consientan con sus decisiones. Esto es, si una empresa es justa, necesariamente los intereses de sus stakeholders deben ser tenidos en cuenta al momento de decidir. Ello implica dos cosas: por una parte, la aplicación del principio de transparencia como tal y, por otra, un enfoque normativo de los stakeholders.

El principio de transparencia, en el ámbito empresarial, refiere en principio a dos componentes: la rendición de cuentas y el acceso a la información. La rendición de cuentas indica que las empresas deben dar cuenta de sus acciones a todos los afectados por ella. Como lo señala García Marza (2004: 240), la rendición de cuentas permite a los ciudadanos evaluar las acciones de las empresas y su ausencia reduce la credibilidad y por tanto la legitimidad y la confianza en las organizaciones. Ahora bien, con respecto a "la apertura y flujo de información al dominio público", esta incluye aspectos como la publicación de los estados contables y financieros, las contribuciones a campañas políticas, las estadísticas comerciales y monetarias, etc. Sin embargo, para las empresas privadas esta exigencia tiene un importante límite: la protección de la propiedad intelectual. En todo caso, la transparencia exige que la información que se divulgue sea veraz, clara, disponible, oportuna y pertinente. Sólo así los agentes afectados por las empresas pueden tener elementos de juicio para evaluar su desempeño.

Respecto del enfoque ético o normativo de la teoría de los stakeholders, del que se hablará con más detenimiento posteriormente, este sostiene que son los intereses moralmente legítimos de los grupos, y no su poder, los que deben prevalecer en la definición de los fines de la empresa. Adoptar esta perspectiva normativa de los stakeholders implica que la empresa, en su proceso de toma de decisiones, debe generar espacios de deliberación en los que los grupos involucrados discutan las razones de sus posibles decisiones. Estas razones, para ser aceptables, no pueden ser razones privadas 
a las que únicamente pueden acceder unos cuantos y no todos los afectados; exige además que todos los stakeholders aduzcan razones que unos y otros puedan compartir.

\subsection{Respecto de la relación con otros valores}

Al comprender la transparencia como un concepto vinculado a la necesidad de justificar las normas que articulan y afectan a todos los miembros de un contexto específico, podemos establecer una relación mucho más clara con otros valores propios de los sistemas de gestión ética empresarial, tales como los derechos humanos, la autonomía o la equidad. En esta sección queremos defender la siguiente idea: la transparencia se fundamenta en otros valores y, al mismo tiempo, es condición de posibilidad para que esos otros valores puedan ser efectivamente realizados.

La transparencia, entendida únicamente como valor, puede concebirse como una idea abstracta acerca de algo que debe ser buscado por las empresas con el fin de obtener un bien ulterior. Pero también puede ser entendida no como un medio para algo más, no como valor abstracto, sino como una serie de prácticas, de relaciones $\mathrm{y}$, especialmente, de actitudes con contenidos sustantivos. Al ser entendida de esta última forma, la transparencia, como otros valores, sólo tiene sentido en su realización práctica, adquiriendo su significado en un contexto relacional entre personas que pertenecen a una colectividad (de aquí su caracterización como moral, como perteneciente a las exigencias que se hacen entre agentes) y que se expresa en una serie de acciones con contenidos específicos, que pueden ser seguidos y evaluados con el fin de asegurar su cumplimiento; de ahí que la construcción de indicadores resulta fundamental.

Como ya se ha indicado, la transparencia se funda en valores como la autonomía, el respeto, la confianza o la honestidad. Algunos ejemplos: la transparencia se relaciona con la forma en que las personas reciben información veraz a partir de la cual pueden tomar decisiones autónomas informadas e impide que puedan ser engañadas al poder tener acceso a las fuentes de la información que se les suministra. Cuando una persona $u$ organización actúa transparentemente está reconociendo que el otro, que la persona o grupo ante el cual está respondiendo, merece respeto y que dicho respeto se traduce en las prácticas de transparencia que se están llevando a cabo. Una actitud transparente por parte de una organización, sus directivos, empleados y, en general, cualquier grupo de interés, promueve la confianza organizacional al crear un ambiente en el que los miembros de la colectividad pueden esperar válidamente que se los trate como agentes moralmente autónomos cuyos intereses se están teniendo en cuenta al 
momento de la toma de decisiones que los afectan. Las prácticas transparentes no sólo coadyuvan a la generación de confianza vertical y horizontal, sino que son necesarias para la toma de decisiones de forma equitativa y justa; en este sentido, se presume que una decisión adoptada transparentemente es más justa y el acceso a las razones tenidas en cuenta para adoptar las decisiones permite que los agentes evalúen por sí mismos la legitimidad de las mismas y, llegado el caso, controvertirlas con razones que sean legítimas. Conocer las razones por las cuales se adopta una decisión, así como asegurar mecanismos adecuados para que todos los afectados puedan participar y vean representados sus intereses indica no sólo un mayor grado de organización empresarial sino, además, que la empresa puede considerarse como una institución moral, lo cual a su vez necesariamente redunda en unos mayores niveles de confianza. De este modo, se crea un circulo virtuoso que solamente puede traer resultados positivos a nivel de eficiencia y productividad; en suma, la transparencia se fundamenta en otros valores, y es necesaria para que estos últimos se realicen.

Sin embargo, de esta centralidad de la transparencia no se deduce que sea la única herramienta en la lucha contra la corrupción. Al considerar la transparencia como el valor único y absoluto, que sirve de guía para la lucha contra la corrupción, corremos el riesgo de desvincularla de otros valores que son más importantes para la vida en común en nuestras sociedades. Ni la ética empresarial, ni la ética de las organizaciones, ni la ética de lo público se reducen a la transparencia. La autonomía, el respeto a la dignidad humana, la libertad, el respeto a los derechos humanos son valores que poseen una importancia superior a la de la propia transparencia. Si bien la transparencia, como ya se indicó, es condición necesaria para la realización de dichos valores, en dicha realización la transparencia es el vehículo para que los ciudadanos y las instituciones configuren sus decisiones y prácticas acordes con principios como la autonomía, el pluralismo, la equidad y la solidaridad.

Un ejemplo de lo anterior es la relación normativa entre transparencia y derechos humanos, en especial, en el contexto empresarial. Los Principios Rectores sobre las Empresas y los Derechos Humanos de las Naciones Unidas establecen las obligaciones que las empresas y corporaciones tienen respecto de la promoción y respeto de los derechos humanos en el ejercicio de su actividad empresarial. Respecto de dichas obligaciones, el principio 15 establece la necesidad de establecer políticas y espacios adecuados para, entre otros, identificar, prevenir, mitigar y hacerse responsable de cómo las actividades empresariales impactan los derechos humanos de los individuos que trabajan en sus organizaciones, así como los de las comunidades en las cuales se desarrolla su ejercicio empresarial (ONU, 2011). Para ello 
se hace necesario que los diversos grupos conozcan las normas de derechos humanos, discutan su lugar en el ejercicio empresarial y laboral particular y puedan ser esgrimidas en las tomas de decisiones que los afectan y afectan a otros. Estas exigencias son aspectos del ejercicio transparente de la actividad empresarial y, a la vez, condiciones para que el respeto a los derechos humanos se garantice de manera efectiva.

Adicional a lo anterior, los principios 16 a 24 desarrollan la forma en que las empresas deben comprometerse en la defensa y promoción de los derechos humanos en su actividad empresarial. Dichos principios se relacionan con la forma en que los derechos humanos solamente pueden tener operativización en la actividad empresarial a través de procesos y prácticas transparentes. Así, por ejemplo, el principio 18 establece la necesidad de que la empresa establezca un proceso de consulta significativa con aquellos grupos de interés cuyos derechos humanos pueden ser potencialmente violados con la actividad empresarial; dicho proceso de consulta debe estar caracterizado por una escucha activa de las razones que los grupos potencialmente afectados proporcionan en el proceso y, más importante aún, en el proceso de deliberación y toma de decisiones debe ser clara la importancia que la protección de los derechos humanos tiene para la empresa en cuestión. Más decisivo aún es el principio 21 que establece la obligación de comunicar públicamente los posibles impactos que la actividad empresarial tiene en la promoción de los derechos humanos o, eventualmente, las posibles violaciones de derechos humanos que pudieren causarse con su actuar; dicha comunicación debe ser, entre otras características, permanente y accesible por cualquier audiencia, y proveer suficiente y adecuada información, relevante para la toma de decisiones en materia de derechos humanos. De este modo, la transparencia (las prácticas transparentes de deliberación) se erige como mecanismo para la implementación de otros valores, el del respeto a los derechos humanos en este caso.

\section{LA TEORÍA DE LOS STAKEHOLDERS Y LA RENDICIÓN DE CUENTAS}

No sobra recordar que las empresas son organizaciones complejas en las que hay conflictos entre los distintos grupos involucrados en su funcionamiento; en este sentido, la transparencia tiene un papel clave en la regulación de las relaciones entre estos grupos. Como veremos a continuación, este valor exige que la empresa, como un todo, en sus decisiones y acciones, tenga en cuenta los intereses moralmente legítimos de quienes son afectados por actividades empresariales.

Para dar cuenta de lo anterior, haremos una breve presentación de la teoría de los stakeholders usada tanto por el GRI como por ETHOS (2.1). 
Posteriormente, discutiremos cuestiones centrales que, según este enfoque, deben tenerse en cuenta en la manera cómo las empresas deben rendir cuentas a los stakeholders: ¿cuáles son los grupos de interés que deben considerarse? (2.2); ¿cuáles intereses legítimos deben tenerse en cuenta? (2.3); y ¿cuál es el papel de los stakeholders en la rendición de cuentas? (2.4).

\subsection{La teoría de los stakeholders}

Las empresas son al mismo tiempo comunidades políticas y comunidades morales. Ellas son espacios sociales en las que no solo hay relaciones de poder, sino que también se desarrollan relaciones interpersonales entre los individuos que hacen parte de ella. Como señalamos anteriormente, hoy en día es un hecho que la empresa tiene un enorme poder sobre los distintos ámbitos de la vida social; y también es un hecho, que al interior de ellas hay conflictos entre distintos actores que en su mayoría son resueltos de acuerdo con la capacidad que tenga cada parte para imponer sus decisiones sobre el resto. La teoría de los stakeholders reconoce esta realidad de la pluralidad de poderes e intereses en la empresa. Para esta concepción, la empresa no es ese todo armónico en el que todos los grupos están sintonizados con los intereses de la empresa. Esto no quiere decir que la empresa sea una especie de estado de naturaleza del que solo se sale a través de la fuerza que tienen los dueños del capital para aplastar los esfuerzos de los otros grupos que se les enfrente como los trabajadores, o la competencia. La empresa también es un ente cooperativo en donde se trabaja mancomunadamente en pro de ciertos objetivos comunes. Lo que caracteriza la teoría de los stakeholders es su idea de que la definición de esos objetivos comunes debe partir del hecho de que al interior de ella hay una pluralidad de grupos con distintos intereses y concepciones acerca de cómo debe funcionar la empresa. Para esta concepción, en sociedades pluralistas y democráticas, como pretende ser la sociedad colombiana después de la constitución del 91, la gestión de las empresas no está solo al servicio de los propietarios de las empresas y sus propios intereses, sino que debe considerar los intereses (stakes) de todos los agentes o grupos que participan en la empresa (propietarios, directivas y trabajadores) o que son afectados por sus acciones (distribuidores, consumidores, proveedores, comunidad, Estado).

Para Edward Freeman y William Evan, dos de los defensores más reconocidos de este enfoque, un grupo de interés o stakeholder es "cualquier grupo o persona que puede afectar o ser afectado por la corporación" (Freeman \& Evan, 1995: 149). De acuerdo con esta definición, poder e interés (la capacidad para afectar y la posibilidad de ser afectado) son los elementos que determinan quién es un stakeholder, cada stakeholder cuenta 
con poderes e intereses que pueden diferir y que, eventualmente pueden generar conflictos. En las últimas décadas, se han dado varios desarrollos de esta forma de ver la empresa. La distinción propuesta entre otros por García-Marzá (2004), Gilbert y Rasche (2008) entre un enfoque estratégico y un enfoque ético de los stakeholders arroja luces para entender mejor la perspectiva que se debe asumir en la rendición de cuentas ${ }^{5}$.

En el enfoque estratégico, los stakeholders se ven solo como medios para los fines de la organización; lo esencial es el poder que tiene el grupo para afectar a la empresa y, por tanto, en esta perspectiva, solo los intereses de los grupos que tienen poder son considerados. Este enfoque puede verse como una versión empresarial de lo que en política se llama realpolitik y que consiste en considerar al poder como el criterio definitorio central en el proceso de toma de decisiones: dado que los fines e intereses de la empresa no surgen espontáneamente, sino que dependen de los grupos con capacidad de influir, y dado que hay diferencias de intereses entre estos grupos, los intereses y fines de la empresa deben ser definidos por las partes con más poder. Lo central en este caso es el juego de poder que se da al interior de la empresa, con sus negociaciones e intercambio de favores. Así, cada uno de los stakeholders, no solo los directivos y propietarios, hace un cálculo estratégico en el que considera los recursos de poder e intereses de cada grupo; con base en este cálculo, cada stakeholders elabora un plan de acción.

Bajo este enfoque, la rendición de cuentas presenta las acciones de la empresa en función de lo que conviene más a los grupos de interés que tengan más control sobre ella. En este caso, la rendición de cuentas es reputacional, pues lo que se busca con ella es darle legitimad a la empresa frente a los grupos de interés afectados. Al mismo tiempo, los grupos de interés que tengan más poder presionaran para que dicha rendición de cuentas no afecte sus intereses. El problema de seguir un enfoque como este es que la rendición de cuentas es más susceptible de ser menos transparente y que no se consideren seriamente las demandas moralmente válidas de aquellos grupos que son afectados por la empresa, pero que no tienen ningún poder.

En oposición al enfoque estratégico, el enfoque ético o normativo de la teoría de los stakeholders sostiene que son los intereses moralmente legítimos de los grupos involucrados los que deben prevalecer en la definición de los objetivos de la empresa. Para esta concepción, en sociedades pluralistas y democráticas, el propósito de la empresa no es sólo satisfacer los intereses de los propietarios de las empresas, sino que debe considerar los

\footnotetext{
5 Estos autores también hablan de un enfoque descriptivo que tiene que ver con dar cuenta sobre cuáles stakeholders tiene la empresa en un momento dado.
} 
intereses (stakes) de todos los grupos que participan en la empresa (propietarios, directivas y trabajadores) o que son afectados por sus acciones (distribuidores, consumidores, proveedores, comunidad, estado).

Como lo señalan Freeman y Evan (1995: 115), los stakeholders no deben verse como meros medios, sino como fines en sí mismos en un sentido kantiano, es decir, como interlocutores que tienen derecho a intervenir cuando sus intereses están siendo afectados. Esta concepción del stakeholders tiene dos implicaciones: en primer lugar, para que los fines de la empresa sean legítimos ellos no pueden ser el resultado del juego de fuerzas al interior de la empresa, sino que deben ser definidos a partir de un proceso de deliberación en los que los distintos grupos afectados puedan participar sin coacciones de ningún tipo y hacer valer sus intereses cuando estos sean legítimos. En segundo lugar, en la identificación de los stakeholders de la empresa se debe considerar como criterio central los intereses afectados y no el grado de poder que tenga el stakeholder.

Estas dos implicaciones no significan que este enfoque ignore la realidad del poder en una empresa. Para este enfoque, el proceso de rendición de cuentas al mismo tiempo que la realidad conflictiva de la empresa, no es un discurso que busca justificar el poder, sino que es una perspectiva crítica que enfatiza los límites morales de su ejercicio en la empresa. Es el respeto a estos límites morales, lo que da la legitimidad moral a la empresa. Esta afirmación supone que el ejercicio injustificado del poder genera desconfianza y a la postre es perjudicial para los mismos intereses de los grupos que tienen poder. Así, por ejemplo, un empresario que a toda costa quiere imponer sus intereses a sus empleados sin tener en cuenta los de ellos, no solo carece de legitimidad frente a ellos, sino que, además, puede generar ineficiencias, pues en la medida en que los empleados sienten que sus opiniones no son tenidas en cuenta y expresarlas puede ser motivo de sanción y despido, se pierde la confianza, y con ello la evaluación crítica de los procesos de gestión necesaria para hacer más eficiente la empresa desaparece. Este caso muestra que el ejercicio del poder sin legitimidad moral puede no solo ser perjudicial para los intereses de la empresa, sino que, además, puede incidir negativamente en la transparencia, ya que cuando hay un ambiente de desconfianza, el intercambio de información se distorsiona.

Adicionalmente, estudios como los de Fransen y Kolk (2007) y de Owen, Swift, Humphrey y Bowerman (2000) muestran que cuando los estándares de auditoría social asumen de manera seria un enfoque normativo se pueden tener varios resultados positivos tanto para la sociedad como para las organizaciones: (i) se mejoran los niveles de confianza de la organización con los stakeholders; (ii) se logra un mayor control por parte de los stakeholders de las políticas y acciones de la organización; y (iii) se da 
un mayor aprendizaje de la organización y de sus stakeholders sobre cómo manejar asuntos como el medio ambiente, la transparencia en la información, las relaciones con la comunidad, el respeto a los derechos humanos, etc.

Ya que el objetivo primordial de este artículo es establecer los procesos que una empresa debe adelantar para ser efectivamente transparente y no meramente considerada como tal con miras al incremento de su reputación frente a otros, adoptaremos este enfoque normativo de la teoría de los stakeholders. Así, en lo que resta, se analizaran tres aspectos de este enfoque que son relevantes en la rendición de cuentas: ¿cómo identificar los stakeholders que se deben tener en cuenta?, ¿cómo establecer cuáles son los intereses o demandas morales legítimas frente a las cuales las empresas tienen que responder y que, a su vez, deben ser el objeto sobre el cual debe centrarse la rendición de cuentas?, y ¿cuál es el rol de los stakeholders en el proceso de rendición de cuentas?

\subsection{Identificación de los stakeholders}

La empresa hace parte del entramado social y por tanto sus acciones tienen efecto sobre una infinidad de grupos. Atender los intereses de todos estos grupos es fácticamente imposible y no puede ser exigible, pues ningún agente moral está obligado a hacer lo imposible. Así pues, se presenta el problema de establecer cuáles son los grupos de interés que se van a considerar como stakeholders. Esta identificación es fundamental para establecer la responsabilidad que tiene la empresa como un todo hacia los stakeholders ${ }^{6}$. Pero, adicionalmente, en tanto todo derecho implica obligaciones, en el caso de las empresas esta afirmación significa que el reconocimiento de los derechos de los stakeholders debe ser algo recíproco. Así, cada stakeholder, en la medida que tiene la pretensión de que sus derechos sean reconocidos, también debe reconocer que la empresa como un todo y los otros stakeholders tienen sus derechos y que él está en la obligación de respetarlos ${ }^{7}$.

Como ya se indicó, el enfoque estratégico tiene como criterio de identificación relevante el poder del stakeholder. Así entre más poder tenga, más

6 La expresión "la empresa como un todo" no hace referencia al conjunto de stakeholders, sino a que la empresa como tal es un ente cooperativo que genera beneficios y que para su funcionamiento requiere de un esfuerzo mancomunado de todos los stakebolders (Goodstein \& Wicks, 2007: 376).

7 Goodstein y Wicks (2007: 375-376) han señalado, con razón, que existe una tendencia entre los defensores de la teoría de los stakeholders a enfocarse en los derechos de los stakeholders y en las responsabilidades de las empresas hacia ellos, sin tomar en cuenta las obligaciones que los stakeholders tienen hacia la empresa. 
relevante es el stakeholder con respecto a los intereses propios de la organización. Para este enfoque estratégico, la rendición de cuentas ética sólo es importante en tanto pueda ser un buen negocio; bajo esta perspectiva, la ética se pone al servicio de la rentabilidad: la rendición de cuentas no tiene una motivación propiamente ética, sino instrumental. Rasche y Esser (2006: 257) sostienen que cuando en las organizaciones se establece el poder como criterio de identificación de stakeholders, la rendición de cuentas es más susceptible de ser manipulada por parte de los stakeholders que tengan mayor control sobre las decisiones de la organización.

Como señalan Donaldson y Preston (1995), la cuestión central en la concepción estratégica de los stakeholders es cómo ellos contribuyen al cumplimiento de los objetivos de la organización, que generalmente se enfocan en la rentabilidad financiera (Rasche \& Gilbert, 2008: 761). Sin embargo, en el enfoque normativo, el asunto es cómo debe responderse a los intereses legítimos de los stakeholders, para lo cual lo primordial es establecer cuáles son estos stakeholders que pueden tener intereses legítimos. Así pues, bajo esta perspectiva, los grupos de interés más relevantes no son los que más influyen, sino los que tienen sus intereses legítimos más afectados por la actividad de la organización.

El enfoque normativo apunta a que los criterios que se deben tener en cuenta para identificar los stakeholders deben ser la legitimidad y urgencia de sus intereses legítimos. Sobre estos intereses legítimos hablaremos en la próxima sección; por ahora, es importante indicar que la rendición de cuentas ética de las organizaciones requiere una evaluación sobre la forma en que en ella se cumple con las responsabilidades legales, sociales, éticas y hasta filantrópicas que le corresponde. Cada una de estas funciones tiene, en principio, un stakeholders que debe cumplirla. Con base en estas funciones es posible realizar una tipología de los stakeholders (Araque \& Montero, 2006: 100-120) que, cualquier organización debe tener como mínimo: el primer tipo refiere a aquellos grupos que participan de la cadena interna de valor: los propietarios, las directivas y los empleados. El segundo tipo refiere a aquellos grupos que no están directamente involucrados en el proceso de producción, pero son fundamentales para que este se dé, porque, o bien, proveen la maquinaria y la materia prima necesaria como es el caso de los proveedores, o bien adquieren el servicio o producto final, como son los distribuidores y consumidores. El tercer tipo corresponde a aquellos grupos que son afectados por las actividades de la organización, pero que no intervienen en el proceso de producción; a este tercer tipo, corresponden las comunidades y el Estado ${ }^{8}$.

\footnotetext{
8 En relación con este último tipo de stakeholders, las empresas como un todo, en un caso como el colombiano, tienen varias responsabilidades: en primer lugar, deben
} 
Para finalizar esta sección, cabe señalar que, ligado al problema de la identificación de los stakeholders, está el asunto sobre cómo escoger a quienes van a representar y defender los intereses del stakeholder en el proceso de rendición de cuentas. Los stakeholders no son grupos homogéneos, dentro de ellos pueden existir algunas facciones que están más cerca a los intereses de las directivas y pueden existir otros más renuentes. Desde un punto de vista estratégico, las directivas pueden estar más interesadas en que los representantes sean más dóciles y que las organizaciones o asociaciones que agrupen a los stakebolders sean lo menos autónomas posibles y que dependan al máximo de la compañía. Es claro que ni la docilidad ni la falta de autonomía de los representantes y asociaciones son propicios para los fines de la auditoria ética, pues ello disminuye sustancialmente el potencial de crítica que debe tener un proceso de rendición de cuentas.

Principios normativos como los derechos humanos, el desarrollo sostenible y la transparencia tocan aspectos sensibles de las organizaciones que deben ser afrontados y cuya solución implican sacrificios, en especial de los directivos e inversionistas, que históricamente han sido los principales beneficiarios en el sistema de mercado. En este sentido, como señala Owen et al. (2000), la rendición de cuentas con una orientación ética, llamada por varios estudiosos, auditoría ética ${ }^{9}$, no fue pensada para complacer a las compañías, sino para causar escozor, para incomodar. En la medida en que los códigos éticos empresariales no exijan en sus procedimientos de implementación que los representantes de los stakeholders deben ser escogidos por quienes van a ser representados y además velar para que no haya interferencia de los otros stakeholders en su elección y representación autónoma, los procesos de auditoría social no serán más que ejercicios de autocomplacencia al servicio de la imagen de la compañía, muy lejos de los instrumentos que deben ayudar a las empresas a responder de manera efectiva y transparente a las demandas legítimas de sus stakeholders.

contribuir con la construcción de una paz duradera y por ese motivo es fundamental la participación de ellas en la reconstrucción del tejido social de las comunidades más afectadas; en segundo lugar, dadas las desigualdades sociales y la debilidad del estado, las empresas tienen la obligación, a través del pago de impuestos, de fortalecer las instituciones estatales a nivel nacional y territorial; en tercer lugar, las empresas deben ser corresponsables de la sostenibilidad ambiental del país, por lo que es necesario que ellas adopten tecnologías limpias y ayuden a mitigar el deterioro ambiental.

9 Para un análisis de lo que significa la auditoria ética y como sinónimo de cierta forma de hacer la rendición de cuentas en las organizaciones, se sugiere consultar el trabajo de Rasche y Esser (2006). 
2.3. Los intereses moralmente legítimos

Los stakeholders pueden tener muchos intereses en juego con respecto a una empresa, pero la empresa no tiene que atender a todos estos intereses, sino sólo aquellos que considera moralmente legítimos. Pero, ¿qué es un interés moralmente legítimo? ¿qué criterios se pueden usar para justificar moralmente un interés de una persona o grupo en relación con una organización?

En principio, estos criterios corresponden a los valores que la empresa adopta: si los intereses del grupo son acordes a esos principios de la empresa, entonces el grupo tiene unos intereses legítimos; esto es, unos derechos frente a lo que la empresa tiene que responder. Pero esto por sí solo no hace que dichos intereses sean moralmente legítimos, sino solo que son legítimos para la empresa en cuestión. Sin embargo, esto no es suficiente, pues a las empresas también se les exige que sus valores y principios sean compatibles con los valores que configuran el ethos compartido de la sociedad en que viven, como pueden, por ejemplo, los derechos humanos. Así, si un grupo tiene un interés legítimo para la empresa en cuestión, y esa empresa efectivamente ha adoptado principios y valores compatibles con los derechos humanos, la conclusión sería que no solamente la empresa es una moralmente legítima, sino, además, que el interés del grupo también lo es. Por otra parte, si la empresa en cuestión realiza actividades que lesionan los derechos humanos de los miembros de un grupo determinado, la empresa no es moralmente legitima, pero tal grupo sí tiene un interés moralmente legítimo frente al cual la organización debe responder apropiadamente.

Un criterio de los derechos humanos es útil para identificar intereses moralmente legítimos, pero no es el único. La ética corporativa tiene que ver con los valores y principios que sigue la empresa como un todo; para que estos valores sean en principio válidos, deben ser asumidos voluntariamente por todos los stakeholders que intervienen en la empresa. En este sentido, no debe confundirse la ética corporativa con las creencias morales de sus propietarios y directivos: si, por ejemplo, los trabajadores y proveedores acatan los valores de la empresa por miedo a ser despedidos o porque no les compran sus productos, para estos agentes dichos valores no tienen validez ética. Para que sean éticamente válidos se ha de recurrir a algún valor o principio que primero sea razonablemente compartido por los grupos involucrados en una organización; valor y principio que debería ser acorde con los principios que se aceptan también razonablemente en la sociedad donde se desenvuelve la empresa. El principio de justificación y su umbral de reciprocidad y generalidad nos proporciona una buena pauta para identificar cuándo un interés tiene validez ética: un interés es 
moralmente legítimo si se tienen en cuenta de manera razonable los intereses de todos, y el interés no es legítimo si solamente tiene en cuenta de manera arbitraria los intereses de alguno o varios de los stakeholders (generalmente los de mayor poder).

Los valores o principios empresariales se expresan en códigos de conducta, que pueden ser escritos o no y que se vuelven parte de su cultura organizacional. Como lo señala Hoffman (1995: 578), un signo de que los empresarios valoran seriamente el punto de vista moral, y no meramente para incrementar su reputación, es adoptar explícitamente códigos, crear mecanismos para hacerlos cumplir, tratar a toda costa de incorporarlos en su cultura organizacional, y rendir cuentas a sus stakeholders. Un signo aún más significativo es que dichos valores sean, además, éticamente válidos: ser asumidos voluntariamente por todos los stakeholders de la empresa. Cualquier empresa puede crear un código de conducta y un sistema de gestión ética que lo efectivice ${ }^{10}$, pero si los valores contenidos en dicho código no son éticamente válidos, la empresa no puede considerarse como moralmente legítima; este es justamente el reto y la dificultad.

De acuerdo con lo dicho, se puede afirmar que los valores de una ética cívica son el criterio central desde el cuál se han de evaluar la pertinencia y validez de los valores que cada organización asume, y determinar hasta qué punto las demandas que en un momento puede hacer alguien afectado por la acción de una empresa es moralmente legítima y por tanto merece una respuesta. Los valores de una ética cívica son éticamente válidos a priori en sociedades liberales y democráticas, ya que no se espera que un grupo los rechace razonablemente; es decir, no se espera que alguien rechace el proteger los derechos humanos, la libertad o la autonomía, por ejemplo.

La rendición de cuentas, en cuanto remite a los valores éticos que la organización asume, se concibe como un sistema de evaluación del comportamiento ético de los distintos grupos de interés que hacen posible el funcionamiento de la organización. Como lo señala Hoffman (1995: 578), las empresas no viven en el vacío; sus valores y principios están determinados en buena parte por las sociedades en que se desenvuelven. Del mismo modo, Adela Cortina (1994) afirma que la ética corporativa es parte de la ética civil, de la ética de los ciudadanos. Ahora bien, en sociedades

10 De hecho, así sucede: una breve revisión de los códigos de ética de varias empresas colombianas muestra una diversidad de declaraciones de principios y valores, así como de mecanismos para que esos valores impregnen las prácticas de la organización. Muchas de estas declaraciones, empero, están llenas de lugares comunes: formulaciones ambiguas en la que todo cabe y que pueden servir de sombrilla para justificar acciones que van en contra de los valores éticos propios de una sociedad democrática respetuosa de los derechos de los ciudadanos. 
democráticas y liberales, los principios normativos que rigen la ética civil, de la que la ética corporativa es parte, son la libertad, la igualdad, la solidaridad y el respeto a la diferencia, y la honestidad y transparencia. Son estos valores mínimos de la ética cívica los que marcan el alcance que debe tener la rendición de cuentas de las empresas: son estos valores, y no los intereses particulares de los grupos afectados, a los que debe atender la rendición de cuentas.

En este sentido se puede decir que, además de la exigencia de ser eficientes, las empresas colombianas deberían construir una cultura organizacional basada en el respeto a los derechos humanos, la transparencia, y la búsqueda del bien común. Estos principios están consagrados en la Constitución de 1991 y pueden ser fundamentados en el citado principio de la ética discursiva según el cual una empresa es justa si las consecuencias directas e indirectas de sus acciones y políticas son aceptadas voluntariamente por sus stakeholders.

\subsection{El papel de los stakeholders en la rendición de cuentas}

Los espacios de participación de los stakeholders contempladas al momento de implementar el sistema de gestión ética son determinantes para que ellos puedan efectivamente ejercer su rol en el proceso de rendición de cuentas. Como lo muestran Fransen y Kolk (2007: 679), un estándar que pretenda ser un instrumento para que la organización responda de manera efectiva a los intereses legítimos de sus stakeholders debe ser exigente con respecto a la participación de estos en el proceso. Así, en los procesos de rendición de cuentas, los representantes de los stakeholders deben ser autónomos con respecto a los otros stakeholders y deben contar con el respaldo de los miembros de su grupo para que no haya manipulación en el proceso de evaluación del comportamiento de la organización. De no cumplirse lo anterior, los stakeholders serían meros invitados para refrendar las decisiones de los directivos o para recolectar información y ejercerían de manera efectiva su papel de guardianes del comportamiento ético de la organización. Más grave aún, si los intereses de los stakeholders no son tenidos en cuenta, la decisión adoptada carece de validez ética al no ser justificable para todos los afectados por ella. Por lo anterior puede decirse que entre más participación se le dé a los stakeholders en el proceso de rendición de cuentas, menos posibilidades de manipulación y sesgo en la evaluación existirá.

Pero ¿cuáles deben ser las actividades de los stakeholders en los procesos de rendición de cuentas? En términos generales, hay cuatro actividades en que ellos pueden cumplir un papel importante: (i) la recolección de la 
información, (ii) el análisis e interpretación de los datos, (iii) el monitoreo y la evaluación, y (iv) la comunicación y la retroalimentación.

Aunque los dos primeros tipos de actividad son claves en la rendición de cuentas, la participación de los stakeholders es pasiva y las interacciones con la organización se pueden limitar a llenar encuestas, participar en grupos focales, dar su opinión de expertos sobre ciertos asuntos e indicadores. Cuando el proceso se queda en esto y se deja en manos de las directivas todo el resto, hay mayores posibilidades de que la organización presente la información de una manera más favorable en términos de imagen corporativa.

La participación independiente de los stakeholders es clave en la etapa de monitoreo y comunicación para garantizar mayor transparencia. Esta etapa consiste básicamente en verificar la calidad de la información suministrada y evaluar el desempeño de la organización a partir de una aplicación adecuada de indicadores diseñados para este propósito. Hay diversas formas de encarar esta etapa: algunas organizaciones lo dejan en manos de las propias directivas, convirtiéndolo en un mero ejercicio de auditoría interna; otras organizaciones acuden a auditores externos. Aunque esta última alternativa genera más confianza a los stakeholders, puede haber algo de manipulación en la medida en que es la administración de la organización la que escoge la compañía que va a auditar y, como señala Owen et. al. (2000: 88), hay una tendencia a escoger auditores externos más "amigables" a la organización. Finalmente, otra alternativa es permitir la participación directa de los stakeholders en el proceso de seguimiento y que ellos mismos, con la ayuda de auditores externos, lleven a cabo esta tarea. Es claro que esta última alternativa ofrece un mayor involucramiento de los stakeholders y mayores oportunidades para que ellos puedan ejercer un rol crítico de manera autónoma.

En la etapa de comunicación y retroalimentación es cuando la organización, propiamente dicha, rinde cuentas de sus actividades a los diferentes stakeholders y a la sociedad en general. La manera como se presenta esta información puede generar una imagen positiva o negativa de la organización; por tal motivo, hay un fuerte interés de las directivas en la divulgación de los resultados de las etapas anteriores. Una forma de evitar injerencias indebidas es que los mismos stakeholders tengan la oportunidad de hacer valer sus opiniones con respecto a los resultados divulgados y que estas sean tenidas en cuenta en las acciones y políticas futuras de la empresa. Adicionalmente, si los resultados son comunicados solamente a algunos de los stakeholders, los distintos grupos afectados no tienen la posibilidad de responder críticamente al informe que se haga para este propósito; en casos como este, la auditoría ética no está contribuyendo con la transparencia, sino que se trata de un ejercicio de ocultamiento de prácticas 
de gestión que pueden ser cuestionables. Así, puede decirse que un proceso de rendición de cuentas estará menos sujeto de manipulación, si tiene reglas estrictas que exijan a las organizaciones permitir la participación efectiva de los stakeholders en el proceso de divulgación de la información.

Ahora bien, la efectiva participación de los stakeholders en las dos últimas etapas implica que ellos hagan parte de los grupos o comités encargados por la organización en la implementación del proceso de auditoria ética. Adicionalmente, para prevenir manipulación, en la conformación del grupo no solo se deben establecer las responsabilidades de cada uno de sus miembros, sino que, para que el proceso sea éticamente válido, en virtud de los principios de generalidad y reciprocidad, todos deben contar con los mismos derechos, principalmente a opinar, participar en la toma de decisiones, y ejercer vetos. En general, cuando las condiciones de participación en el proceso de implementación del estándar son equitativas, hay una mayor garantía de que el proceso y los resultados de la auditoría serán imparciales.

3. TRANSPARENCIA Y RENDICIÓN DE CUENTAS EN LOS ESTÁNDARES ÉTICOS

El objetivo central de esta sección es establecer unos mínimos de transparencia que deben ser considerados en el proceso de rendición de cuentas por parte de las empresas que tengan la pretensión de ser organizaciones justas, esto es, organizaciones que defiendan los valores de la ética civil que están a la base de un estado social de derecho, como son, entre otros, los de libertad, equidad y solidaridad. Estos mínimos no se establecen a través de estudios empíricos sobre cómo las organizaciones están de hecho rindiendo cuentas en distintas partes del mundo; la razón de ello es que tal como se llevan a cabo muchos de estos procesos se encuentran procedimientos y prácticas que hacen que ellos no sean transparentes. Precisamente, para saber qué es un proceso de rendición de cuentas transparente se hizo necesario aclarar, previamente y a partir del concepto mismo de transparencia, cuáles son los requerimientos o criterios mínimos que dichos procesos deben cumplir para satisfacer el valor de la transparencia; las meras descripciones empíricas no nos dicen nada sobre estos criterios que son esencialmente normativos.

Esto no quiere decir que lo que se ha propuesto parta de una concepción de empresa desligada de la realidad. Como telón de fondo se tienen dos iniciativas internacionales sobre rendición de cuentas que han sido muy influyentes en Colombia y América Latina en general: el Global Reporting Initiative (GRI) y el Instituto Ethos de empresa y responsabilidad social 
(ETHOS). Dichas iniciativas promueven valores afines a una comprensión de la transparencia asociada a la ética cívica, a saber: el respeto a los derechos humanos, la equidad y la justicia social, la lucha contra la pobreza, la autonomía, y la sostenibilidad ambiental, entre otros. Así, la realidad de que una extensa cantidad de empresas hayan tomado como referentes estas iniciativas es indicio de que, para dichas empresas, estos valores recogen lo que se considera debe ser una empresa justa. En este sentido, si bien los mínimos propuestos no se basan en estudios empíricos, no son ideales irrealizables; lo que más bien expresan estos mínimos, es el horizonte o ideal que debe guiar las decisiones y acciones de una empresa, si esta quiere realmente ser justa. Para entender mejor el argumento, se puede traer lo que pasa con la eficiencia, este también es un ideal normativo que la empresa asume si quiere ser eficiente: que la empresa no sea eficiente no implica que el ideal de eficiencia deba eliminarse, solo que hay que mejorar los procesos que se están utilizando para lograr la eficiencia.

El GRI y ETHOS son guías que orientan a las empresas sobre cómo deben tratar a sus stakeholders, proporcionando pautas sobre cómo seleccionar los stakeholders que participarán en el proceso de auditoría ética, y cuál debe ser su rol en dicho proceso. Ambas iniciativas tienen falencias y fortalezas.

La principal falencia es que ninguna de las dos iniciativas explica claramente cómo deben seleccionarse los representantes de los stakeholders; esto tiene el riesgo de que quien siga el estándar considere que es transparente dejar en manos del equipo que diseña el sistema de auditoria ética establecer el proceso de selección de los representantes de los stakeholders. Pero, esto puede dar lugar a que aquellas empresas más interesadas en su imagen corporativa que en convertirse en empresas justas, busquen formas de seleccionar voceros de los stakeholders que sean más dóciles y más susceptibles a apoyar los intereses de los stakeholders más influyentes y menos dispuestos a defender los intereses de otros stakeholders. Así, la ausencia en el GRI y el ETHOS de exigencias a las empresas en relación con garantizar que quienes actúen en nombre de los stakeholders sean independientes y elegidos por el grupo de interés que representan, puede dar cabida a que una organización use el nombre de estas iniciativas de manera estratégica y trate de ofrecer una imagen moralmente limpia cuando sus procesos no son éticamente válidos.

En cuanto a sus fortalezas, se pueden mencionar varias. En primer lugar, ambas iniciativas coinciden en que los principios y valores de una organización deben ser creados con la participación de grupos representativos de todos los stakeholders directamente relacionados con la organización. En segundo lugar, las dos iniciativas convergen en que tanto el grado 
de influencia de un stakeholder como la magnitud de los efectos de la organización sobre este son criterios de identificación de los stakeholders; así, es claro que combinan un enfoque estratégico con un enfoque normativo de los stakeholders.

En tercer lugar, en el GRI y en menor medida en ETHOS, encontramos una serie de directrices que muestran de manera concreta cómo se debe llevar a cabo un proceso de rendición de cuentas que garantice la participación transparente de los stakeholders. En concreto, en el GRI hay tres principios que señalan las condiciones de participación transparente de los stakeholders en el proceso de auditoría: la inclusividad, la relevancia y materialidad, y el equilibrio. Estos principios, como explicaremos a continuación, son derivaciones de los principios de razonabilidad y justificación ya indicados cuando nos referimos a la relación entre transparencia y razón pública.

El principio de inclusividad: exige a las organizaciones que expliquen cómo se ha dado respuesta a los intereses legítimos de los stakeholders. Para ello, la guía indica que la organización debe utilizar formas de participación que garanticen que las opiniones de los stakeholders "se puedan comprender de manera adecuada" (Global Reporting Iniciative, 2013: 11). La inclusividad, entonces, implica que la rendición de cuentas se debe hacer a través de un proceso dialógico en el que participen todos los stakeholders afectados. Esto no es diferente a lo que la ética discursiva proclama: los participantes afectados por una decisión deben tener la capacidad de proporcionar argumentos entendibles por todos los participantes; argumentos que no puedan comprenderse de manera adecuada implicarían que un interés privado, y no el interés general, se encuentra fundamentando el argumento propuesto.

El principio de relevancia y materialidad se refiere a los asuntos o intereses que deben ser considerados. La relevancia de un asunto está dada por lo que las partes consideren "razonablemente importante a la hora de influenciar las decisiones de la organización y de los stakeholders" (Global Reporting Iniciative, 2013: 13); así, no todos los asuntos deben ser considerados, sino solo aquellos que sea lo suficientemente importantes para todos los involucrados. A su vez, materialidad se refiere a que los asuntos que afectan a los participantes son aquellos que deben ser discutidos por los participantes; ninguna decisión que afecte a alguien puede estar justificada si esa persona no ha participado efectivamente en su discusión. Se podría objetar que estos principios son demasiado vagos para ser usados cuando debe determinarse si un asunto es relevante. Frente a esto, se puede señalar lo siguiente: la aplicación de un criterio como el de razonabilidad para definir la relevancia de una situación, al menos en cuestiones éticas y políticas, no se resuelve de manera unilateral; solo a través de un 
proceso de diálogo es que las partes interesadas pueden llegar a un acuerdo acerca de cuáles asuntos son relevantes o no. A su vez, solamente cuando un asunto ha sido establecido como relevante, esto es, que afecta a los que lo han establecido como tal es que esos mismos afectados pueden discutir acerca de la mejor forma de abordar el asunto. Si un tema no es considerado relevante sino sólo por una persona, no tiene sentido que todos lo discutan; pero si ese asunto es relevante para todos, todos deberían discutir la forma de afrontar ese asunto relevante. Así, si el tema del incremento salarial es un asunto que es considerado relevante por todos los trabajadores de una empresa, así como por las directivas, empleadores y dueños de esta, son todos ellos quienes deberían poder discutir la mejor forma de afrontar el problema.

El principio del equilibrio indica que la presentación sobre cualquier asunto relevante "debe incluir los resultados tanto favorables como desfavorables y cubrir aquellas cuestiones de importancia que puedan influir sobre las decisiones de los stakeholders en proporción a su materialidad" (Global Reporting Iniciative, 2013: 30). Este principio no puede ser entendido de otra forma diferente a la aplicación de la transparencia. Recordemos la definición dada al respecto por Transparencia Internacional (2018): transparencia es iluminar las reglas, planes, procesos y acciones, y saber por qué, cómo, qué y cuánto, asegurando que los funcionarios públicos, directivos, y empresarios actúen razonable y visiblemente, reportando sus actividades. Así, se señala una regla para la organización que indica que la interpretación y la presentación de la información no puede hacerse de manera sesgada, para así no favorecer de manera indebida algunos intereses. Una regla como esta podría ser usada por los distintos stakeholders, sobre todo los más débiles, para exigirle a la organización que en el proceso de rendición de cuentas considere debidamente sus opiniones en la medida en que sean razonables.

Finalmente, para asegurar que estos mínimos de transparencia se cumplan, se hace necesario contar con una batería de indicadores que permita evaluar su cumplimiento. Como bien lo señala Víctor Manuel Quintero, los indicadores son "los criterios que permiten valorar y evaluar el comportamiento de las variables" (1994: 28). En una orientación similar, Nirenberg, Brawerman y Ruiz, señalan que un indicador "es un medida o apreciación, directa o indirecta, de un evento, una condición, una situación, o un concepto" (2003: 154). Ahora bien, la mera presencia de un conjunto de indicadores no implica que la empresa sea transparente: bien puede darse el caso que los indicadores estén siendo usados para incrementar la reputación ética de la empresa sin que ello se reporte en un cambio de la cultura organizacional. Por ello, en la formulación de los indicadores, la transparencia debe tener un puesto preeminente. 
Para que los indicadores sirvan a los propósitos de la gestión ética y no solamente al mejoramiento de la reputación, ellos deben tener una serie de atributos o requisitos que no son solo de índole metodológico, sino también y fundamentalmente normativo; estos atributos son, en el fondo, una concreción del principio de la transparencia. A este respecto, siguiendo los planteamientos de Niremberg et al. (2003: 159), un indicador ético social debe suministrar información que registre de la manera más fiel posible lo que sucede en la realidad de la organización. Para este efecto, en la formulación del indicador se debe especificar el tipo de fuente o método de recolección que se va a emplear en la captura de los datos. Entre más fiable sea el método de recolección y más accesible sean los datos que necesite el indicador, mayor será la validez del indicador. Además, el indicador debe poder ser aplicado por diferentes personas arrojando los mismos resultados, claro está, si se emplearon los mismos procedimientos. De este modo, el indicador debe ser confiable, pues si cada vez que cada una de las partes aplica el indicador arroja resultados distintos frente a los mismos hechos, no será posible llegar a un acuerdo sobre lo que está ocurriendo.

El hecho de que un indicador sea confiable y suministre una información fidedigna no garantiza per se la transparencia, pues es posible que este oculte cambios relevantes. Por ello, el indicador debe ser, adicionalmente, sensible: "ser capaz de registrar los cambios que se producen en la cuestión [...] analizada, por más leves que sean" (Niremberg et al., 2003: 160). Un indicador que no haga visible ciertos cambios puede hacer que las demandas de algunos stakeholders puedan ser consideradas por otros stakeholders como no legitimas en virtud precisamente de que ignoran estos cambios.

Por otra parte, desde la perspectiva normativa de los stakeholders, la información debe estar dirigida al público en general y no a un grupo de especialistas; por tal motivo, un indicador debe ser claro, esto es, que debe ser fácil de interpretar sin caer en la ambigüedad (Niremberg, et al., 2003: 161). El uso de una jerga que solo los iniciados o expertos entienden es una barrera seria para que haya una comunicación efectiva entre los distintos grupos afectados por una organización. En este sentido, la claridad exige traducir el lenguaje especializado de distintas disciplinas que se esté usando en la elaboración de los índices al lenguaje común, sin que ello implique una pérdida en el significado y alcance del indicador.

Otro requisito relacionado con la presentación de un indicador tiene que ver con su función de servir de criterio para evaluar. Un indicador es pertinente sólo si ofrece elementos de juicio para evaluar si las prácticas de la organización expresan o no los principios normativos que dice defender. Esto quiere decir que el indicador, además de registrar unos acontecimientos, de medir unos cambios, debe tener criterios para determinar 
si lo que está ocurriendo es malo, regular, aceptable o bueno. Dichos criterios no pueden quedar a merced de las opiniones de quien aplica el indicador, sino que estos deben ser justificados a partir de los principios normativos adoptados.

Así, en términos generales, se puede decir que un indicador que cumpla con los requisitos de validez, confiabilidad, sensibilidad, claridad y pertinencia puede ser un instrumento valioso para que se propicien procesos de auditoria ética transparentes, esto es, escenarios en donde las posibilidades de manipulación por parte de los stakeholders han sido restringidas.

\section{OBSERVACIÓN FINAL}

A lo largo de este artículo, hemos argumentado que la transparencia en el ámbito empresarial debe apuntar a garantizar que las organizaciones respondan a los intereses moralmente legítimos de los grupos afectados por sus actividades. Para que la rendición de cuentas, que es inherente a la transparencia, no se quede en un discurso dirigido a defender la imagen de la empresa y en proteger los intereses de los grupos de interés más poderosos, es fundamental, que en ella participen de manera efectiva, los grupos afectados. Para que estos procesos no sean susceptibles de manipulación, se requiere, por una parte, que quienes representan a los grupos de interés, sean independientes, tengan representatividad y tengan voz y voto en todas las partes de la evaluación ética de la organización, y no sólo en la recolección y análisis de la información. Finalmente, para que el diálogo sobre el cual debe hacerse la rendición de cuentas no se quede en planteamientos genéricos, es necesario que este se haga con evidencias, expresadas en indicadores, que sean accesibles a todos los grupos de interés, que sean pertinentes, y que den los suficientes elementos de juicio de tal manera que se garantice una deliberación informada, no sesgada y no excluyente entre los grupos de interés que participan del proceso.

\section{REFERENCIAS}

Araque, R. \& Montero, J. M. (2006). La responsabilidad social empresarial a debate. Barcelona: Icaria Editorial.

Coase, R. (1937). The Nature of the Firm. Economica, 4(16), 386-405.

Coase, R. (1960). The Problem of Social Cost. Journal of Law and Economics, 3, 144.

Cortina, A. (1994). Ética de la empresa. Madrid: Editorial Trotta.

Dahlman, C. (1979). The Problem of Externality. Journal of Law and Economics, 21(2), 141-162. 
Dewey, J. (1951). Creative democracy: the task before us. En M. H. Fish (ed.), Classic American Philosophers (pp. 389-394). New York: Appleton-Century Crofts.

Donaldson, T. \& Preston, L. E. (1995). The stakeholder theory of the corporation: concepts, evidence, and implications. The Academy of Management Review, 20(1), 65-91.

Entrepreneur (2017). How Transparency Became a Top Priority for Businesses, and Why You Should Care. Disponible en https://www.entrepreneur.com/article/295739

Forbes (2018). Por qué es importante la transparencia en las empresas. Disponible en http:/ / forbes.es/business/42052/por-que-es-importante-la-transparencia-en-las-empresas/

Forst, R. (2011). The Right to justification. Elements of a Constructivist Theory of Justice. New York: Columbia University Press.

Forst, R. (2014). Justification and critique. Cambridge: Polity Press.

Fransen, L. W. \& Kolk, A. (2007). Global rule-setting for business: A critical analysis of multi-stakeholder standards. Organization, 14(5), 667-684.

Freeman, R. \& Evan, W. (1995). A stakeholder theory of the modern corporation-Kantian capitalism. En M. Hoffman \& R. Frederick (eds.), Business Ethics: Readings and cases in corporate morality (pp. 145-154). Englewood, NJ.: Prentice Hall.

García-Marza, D. (2004). Ética empresarial: Del diálogo a la confianza. Madrid: Editorial Trotta.

Gilbert, D. \& Rasche, A. (2008). Opportunities and Problems of Standardized Ethics Initiatives - a Stakeholder Theory Perspective. Journal of Business Ethics, 82(3), 755-773.

Global Reporting Initiative (2013). Sustainability reporting guidelines. G4. Amsterdam, Netherlands. Disponible en https://www.globalreporting.org/resourcelibrary/GRIG4-Part1-Reporting-Principles-and-Standard-Disclosures.pdf

Goodstain, J. \& Wicks, A. (2007). Corporate and stakeholder responsibility: Making business ethics a two way conversation. Business Ethics Quaterly, 17(3), 375-398.

Hoffman, W. M. (1995). A blueprint for corporate ethical development. En M. W. Hoffman \& R. E. Fredrick (eds.), Business ethics: readings and cases in corporate morality (pp. 557-584). New York: McGraw Hill.

Kant, I. (2018). Hacia la pazperpetua (or. de 1796). Madrid: Ediciones Alamanda. Lozano, J. M. (1999). Ética y empresa. Madrid, Editorial Trotta.

Mundo Ejecutivo (2017). ¿De qué sirve la transparencia en mi empresa? Disponible en http://mundoejecutivo.com.mx/management/2017/04/25/qusirve-transparencia-mi-empresa.

Niremberg, O., Brawerman, J. \& Ruiz, V. (2003). Programación y evaluación de proyectos sociales. Barcelona: Paidós. 
Organización de Naciones Unidas [ONU] (2011). Principios Rectores sobre empresas y derechos humanos. Disponible en https://www.ohchr.org/Documents/Publications/GuidingPrinciplesBusinessHR_SP.pdf

Owen, D., Swift, T., Humphrey, C., \& Bowerman, M. (2000). The new social audits: Accountability, managerial capture or the agenda of social champions? European Accounting Review, 9(1), 81-98.

Quintero, V. M. (1994). La construcción y utilización de los indicadores sociales: una propuesta metodológica. Revista Economía, 18(2), 27-56.

Rascher, A. \& Esser, D. (2006). From stakeholder management to stakeholder accountability. Journal of Business Ethics, 65(3), 251-267.

Rawls, J. (1971). A theory of justice. Cambridge, MA: Harvard University Press.

Rawls, J. (1999). Kantian constructivism in moral theory. En J. Rawls, Collected Papers (pp. 303-358). Cambridge: Cambridge University Press.

Smith, J., Haniffa, R. \& Fairbrass J. (2011). A conceptual framework for investigating 'capture' in corporate sustainability reporting assurance. Journal of Business Ethics, 99(3), 425-439.

Transparency International. The Global Coalition Against Corruption (2018). What is corruption? Disponible en https://www.transparency.org/what-is-corruption\#what-is-transparency

Ulrich, P. (1987). Transformation der ökonomishen vernunf. Stuttgart: Haupt.

Sumario: Introducción; 1. Transparencia; 1.1. Respecto de la idea de razón pública; 1.2. Respecto de la relación con otros valores; 2. La teoría de los stakeholders y la rendición de cuentas; 2.1. La teoría de los stakeholders; 2.2. Identificación de los stakeholders; 2.3. Los intereses moralmente legítimos; 2.4. El papel de los stakeholders en la rendición de cuentas; 3. Transparencia y rendición de cuentas en los estándares éticos; 4 . Observación final; Referencias. 